\title{
Pseudotumor Cerebri Due to Tuberculosis: A Case Report
}

\author{
Maliheh Metanat ${ }^{1}$; Mahboobeh Aghaei ${ }^{1}$; Batool Sharifi Mood ${ }^{1, *}$; Hamideh Mirshekari ${ }^{1}$; \\ Nahid Sepehri Rad ${ }^{1}$; Sahar Reyhani ${ }^{1}$ \\ ${ }^{1}$ Infectious Diseases and Tropical Medicine Research Center, Boo-Ali Hospital, Zahedan University of Medical Sciences, Zahedan, IR Iran \\ ${ }^{*}$ Corresponding author: Batool Sharifi Mood, Infectious Diseases and Tropical Medicine Research Center, Boo-Ali Hospital, Zahedan University of Medical Sciences, Zahedan, IR Iran. \\ Tel:+98-5413228101, Fax: +98-5413236722, E-mail: batoolsharifimood@yahoo.com \\ Received: January 16, 2015; Accepted: January 28, 2015
}

\begin{abstract}
Introduction:Pseudotumor cerebri(PTC) happens when the intracranial pressure increases for no obvious reason. But, some medication, metabolic and collagen vascular diseases such as lupus can cause pseudotumor cerebri.

Case Presentation: Here, we presented a 14-year-old girl who referred to our hospital for fever, headache, vomiting and diplopia which has not respond to antibiotic therapy for acute meningitis. Finally, with more evaluations, we found that she had tuberculous Meningitis(TB).

Conclusions: Although, the exact cause of pseudotumor cerebri in most individuals is unknown, but we should remind tuberculosis specially in endemic area for this infection.
\end{abstract}

Keywords: Intracranial Pressure; Pseudotumor Cerebri; Tuberculosis

\section{Introduction}

The brain and spinal cord are surrounded by cerebrospinal fluid which protect these vital tissues from injury. This fluid is produced in the brain and absorbed into the bloodstream $(1,2)$. The increased intracranial pressure can be a result of a problem in this absorption process. The intracranial pressure increases when the contents of skull exceed its capacity. For example, a brain tumor can increase intracranial pressure so, there's no capacity for the tumor. Several studies indicate that many people with pseudotumor cerebri have stenosis in two large sinuses in the brain (1-6). Some medication (Excess vitamin A and Tetracycline, cyclosporine), metabolic diseases (Addison, Uremia), and collagen vascular diseases such as lupus and behcet's syndrome can cause pseudotumor cerebri $(1,2,4,6)$. Infection is rare cause for pseudotumor cerebri, but fungal diseases and TB which increase intracranial pressure due to sinus stenosis can lead to this condition (3-5). Signs and symptoms of Pseudotumor cerebri include: moderate to severe headaches that may originate behind the eyes and worsen with eye movement, nausea, vomiting, dizziness, diplopia, and photopsia. Some people with pseudotumor cerebri experience progressively worsening vision and may become blind (1, 7-10). Here, we presented a 14-year-old girl who referred to our hospital in Zahedan (Southeastern Iran) for fever, headache, vomiting, and diplopia which has not respond to antibiotic therapy for acute meningitis.

\section{Case Presentation}

In September 2014, a 14-year -old girl form Chabahar in the Southeastern Iran referred to our hospital because of fever, headache, vomiting, and diplopia. Her manifestations start with fever, vomiting and headache. She has been treated with ceftriaxone and vancomycin and acyclovoir for acute meningitis, but she had not any response to treatment and diplopia was added to previous findings then referred to our hospital for more evaluations and rule out of cerebral venous sinus thrombosis. She had no history of recent travel and medication .All signs and symptoms had been occurred during the last two weeks. Her headache was worse at night. Fundoscopy showed papilledema, grade IV. Brain computed tomography scaning (CT-Scan), brain magnatic resonance imaging (MRI) and magnatic resonance venography (MRV) were normal. Cerebral venous sinus thrombosis and so other lesions was rejected. Lumber puncture (LP) was done and these findings were reported: White blood cell;

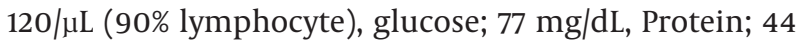
$\mathrm{mg} / \mathrm{dL}$, and the CSF pressure were $45 \mathrm{~cm} \mathrm{H20.} \mathrm{Chest} \mathrm{X-}$ Ray was normal and biochemical test and thyroid and kidney functional tests were within normal ranges. All blood and urine cultures were negative. Diagnostic tests for rheumatologic diseases were negative. Abnormal peripheral visual field was observed in perimetry. The polymerase chain reaction (PCR) on CSF for TB was reported and it was positive for TB. Then, patient treated with anti- 
tubercolous drugs and acetozolamid. LP was repeaded the next days to decrease CSF pressure. Papilledema decreased to grade III. Three weeks later, she was in good condition and diplopia was cured.

\section{Discussion}

Pseudotumor cerebri or idiopathic intracranial hypertension is a disorder of elevated spinal fluid pressure in the brain. PTC is an important condition which should be recognized as soon as possible, because it may lead to progressive loss of vision and permanent blindness $(1,2$, 6). Disease tends to affect overweight people, especially after recent weight gain, even during pregnancy. Certain medications can predispose people to this condition (tetracycline, steroids, and excess vitamin A) $(1,6)$. Some people may be predisposed to PTC because of a congenital defect such as being born with a narrowed vein that drains blood from the brain. There are few reports on infections and PTC. Akhondian et al. from Iran reported a case with PTC due to brucellosis (3). In 2005, Chanet et al. reported a case with PTC due to TB (4). In this case, neuroimaging was consistent with a glioblastoma but, the definite diagnosis was a tuberculoma. Gilad et al. in 2014 also revealed the relation between PTC and varicella (5). Patients with PTC suffer from headache, vomiting, and eye disturbance. Our patient complained from fever, headache, vomiting, and diplopia. Headaches are often at the back of the head, and tend to be worse at night or in the morning (3-5). An eye exam may reveal papilledema. Brain MRI and CT scan may be normal or may show narrowed draining veins or indirect signs of abnormally elevated spinal fluid pressure. Because patients with this condition clearly have signs and symptoms of elevated pressure in the head which is also seen in large brain tumors, but have normal imaging tests, this condition has been named pseudotumor cerebri $(1,4,9)$. Once, the imaging tests have ruled out any tumors or other abnormalities, the pressure of the spinal fluid needs to be measured preferably, by a spinal tap in order to verify the diagnosis. Some patients temporary, relief of their symptoms after drainage of the spinal fluid. When the diagnosis of PTC is made, a careful eye exam and testing of the visual fields should be performed to determine the risk of vision loss (1-4). There are several treatment options, including medications to reduce the spinal fluid pressure or repeated lumbar punctures to keep the pressure down to a safe level $(1,7$, 9). Sometimes, weight loss, can cure the condition. Sometimes, the sheath surrounding the optic nerves is surgically opened with small holes to relieve pressure and prevent deterioration of vision. In severe cases, there is need a shunt that drains the spinal fluid out of the brain. A new option for treatment of PTC is the placement of a stent that is placed across the narrowed portion of the affected vein, allowing blood to drain more freely and relieving fluid pressure in the brain (9).

Although, the exact cause of pseudotumor cerebri in most individuals is unknown, but we should remind tuberculosis specially in endemic area for this infection. In this region, every patient with clinical appearance of PTC must evoke a suspicion for TB.

\section{References}

1. Dave SB, Subramanian PS. Pseudotumor cerebri: an update on treatment options. Indian J Ophthalmol. 2014;62(10):996-8.

2. Blasco Morente G, Tercedor Sanchez J, Garrido Colmenero C, Martinez Garcia E, Molina-Carballo A. Pseudotumor cerebri associated with cyclosporine use in severe atopic dermatitis. Pediatr Dermatol. 2015;32(2):237-9.

3. Akhondian J, Ashrafzadeh F, Beiraghi Toosi M, Hashemi N. A Rare presentation of neurobrucellosis in a child with Recurrent transient ischemic attacks and pseudotumor cerebri (A case report and review of literature). Iran J Child Neurol. 2014;8(2):65-9.

4. Chanet V, Baud O, Deffond D, Romaszko JP, Beytout J. Pseudotumor presentation of intracerebral tuberculomas. South Med J. 2005;98(4):489-91.

5. Gilad O, Shefer-Averbuch N, Garty BZ. Primary varicella infection presenting with headache and elevated intracranial pressure. $J$ Child Neurol. 2015;30(6):793-5.

6. Molobe LT, Shifa J, Silava C, Mojela K, Cainelli F, Vento S. Primary pseudotumor cerebri syndrome in a young obese African woman. Isr Med Assoc J. 2014;16(9):595-6.

7. Kumpe DA, Bennett JL, Seinfeld J, Pelak VS, Chawla A, Tierney M Dural sinus stent placement for idiopathic intracranial hypertension. J Neurosurg. 2012;116(3):538-48.

8. Falardeau J, Lobb BM, Golden S, Maxfield SD, Tanne E. The use of acetazolamide during pregnancy in intracranial hypertension patients. J Neuroophthalmol. 2013;33(1):9-12.

9. Abubaker K, Ali Z, Raza K, Bolger C, Rawluk D, O'Brien D. Idiopathic intracranial hypertension: lumboperitoneal shunts versus ventriculoperitoneal shunts--case series and literature review. $\mathrm{Br}$ J Neurosurg. 2011;25(1):94-9.

10. Mallery RM, Friedman DI, Liu GT. Headache and the pseudotumor cerebri syndrome. Curr Pain Headache Rep. 2014;18(9):446. 